\title{
Discordant associations of lipid parameters with albuminuria and chronic kidney disease: a population-based study
}

Kan Sun ${ }^{\dagger}$, Diaozhu Lin ${ }^{\dagger}$, Feng Li, Chulin Huang, Yiqin Qi, Shengneng Xue, Juying Tang, Chuan Yang, Yan Li, Meng Ren ${ }^{*}$ and Li Yan

\begin{abstract}
Background: Although dyslipidemia is related to the pathogenesis of renal insufficiency, which routinely available lipid measure is more applicable in estimation of kidney function is still uncertain. Our objective was to evaluate inconsistent associations of lipid profiles with both albuminuria and chronic kidney disease (CKD).

Methods: We performed a population-based study in 9730 subjects aged 40 years or older. Definitions of abnormalities in albumin excretion were according to the latest guidelines of American Diabetes Association's Standards of Medical Care. CKD was defined as estimated glomerular filtration rate (eGFR) $<60 \mathrm{~mL} / \mathrm{min}$ per $1.73 \mathrm{~m}^{2}$ or the presence of albuminuria.

Results: There were 2274 (23.4\%) participants categorized as low-grade albuminuria, 639 (6.6\%) participants categorized as increased urinary albumin excretion and 689 (7.1\%) participants categorized as CKD. Triglycerides (TG), high-density lipoprotein cholesterol (HDL-C), Non HDL-C to HDL-C ratio, TG to HDL-C ratio were significantly correlated with urinary albumin to creatinine ratio $(A C R)$, serum creatinine and eGFR (all $P<0.0001$ ). Compare with other lipid parameters, TG to HDL-C ratio have shown the strongest correlation with increased odds of both increased urinary albumin excretion and CKD. No significant associations between lipid parameters and low-grade albuminuria were observed after adjustments for potential confounding factors.

Conclusion: Our study lends support to discordant associations of lipid parameters with albuminuria and renal function. TG to HDL-C ratio is a better marker than other routine lipid measures for identifying renal insufficiency and should be given more consideration in the clinical practice.
\end{abstract}

Keywords: Dyslipidemia, Low-grade albuminuria, Increased urinary albumin excretion, Chronic kidney disease, TG to HDL-C ratio

\section{Background}

In recent years, chronic kidney disease (CKD) has become an important public health problem and directly affects the global burden of death caused by cardiovascular diseases (CVD) [1, 2]. Increased urinary albumin excretion is including historical micro- and macro-albuminuria, which is defined as the urinary albumin-to-creatinine ratio (ACR) ranges greater or equal than $30 \mathrm{mg} / \mathrm{g}$ [3]. Studies

\footnotetext{
* Correspondence: renmeng80@139.com; hfxyl@163.net

${ }^{\dagger}$ Equal contributors

Department of Endocrinology, Sun Yat-sen Memorial Hospital, Sun Yat-sen University, 107 Yanjiang West Road, Guangzhou 510120, People's Republic of China
}



(C) 2015 Sun et al. Open Access This article is distributed under the terms of the Creative Commons Attribution 4.0 International License (http://creativecommons.org/licenses/by/4.0/, which permits unrestricted use, distribution, and reproduction in any medium, provided you give appropriate credit to the original author(s) and the source, provide a link to the Creative Commons license, and indicate if changes were made. The Creative Commons Public Domain Dedication waiver (http://creativecommons.org/publicdomain/zero/1.0/) applies to the data made available in this article, unless otherwise stated.

conducted over the past decades have provided substantial evidence that increased urinary albumin excretion is a risk factor for diabetic nephropathy and cardiovascular diseases [4-6]. Recent studies indicated that low-grade albuminuria (ACR less than $30 \mathrm{mg} / \mathrm{g}$ ) is associated with abnormal cardiac mechanics and might also increase the risk of cardiovascular morbidity and mortality [7-9].

As a modifiable risk factors for cardiovascular disease, controlling of dyslipidemia is one of the most effective way for preventing atherosclerotic cardiovascular disease (ASCVD) in subject with chronic renal insufficiency [10-12]. Atherosclerotic renovascular disease (ARVD) is a fairly common disorder in elderly people [13]. 
However, based on current evidences, the association between dyslipidemia and ARVD is still not confirmed [14, 15]. There is ongoing debate regarding the importance of lipoprotein subclasses and other related markers of lipoprotein metabolism. Guidelines for the detection and treatment of high cholesterol recommend using low density lipoprotein cholesterol (LDL-C) as the primary marker to guide therapy. In addition to LDL-C, Non-high density lipoprotein cholesterol (NonHDL-C), Non-HDL-C to HDL-C ratio (Non-HDL-C/ HDL-C) and triglyceride to HDL-C ratio (TG/HDL-C) are routinely available from the standard lipid profiles. Growing evidences suggests that Non-HDL-C may provide a more accurate measure of coronary heart disease risk than LDL-C [16]. However, in patients with type 2 diabetes, Non-HDL-C/HDL-C is better than Non-HDL-C to predict coronary heart disease [17]. Based on the above arguments, as ASCVD, ARVD and CKD sharing similar pathogenesis and common risk factors, we speculated that the inconsistent association of lipid parameters with vascular might also existed in renal insufficiency.

Previously existing literature and primary analyses have not been implemented to make a systematic comparison on the association of different lipid markers with both of the albuminuria and CKD. In practice, however, it is unclear which routinely available lipid measure is better for the identification of kidney function. Accordingly, we assessed the associations of all routine lipid measures with both albuminuria and renal function in a community-based population.

\section{Methods}

\section{Study population and design}

We performed a cross-sectional study in a community in Guangzhou, China from June to November, 2011. The study population was from the Risk Evaluation of cAncers in Chinese diabeTic Individuals: A lONgitudinal (REACTION) study, which was been set up as a multicenter prospective observational study with the aim of evaluating chronic diseases in the Chinese population $[18,19]$. During the recruiting phase, a total of 10,104 residents aged 40 years or older were invited to participate by examination notices or home visits. In total, 9916 subjects signed the consent form and agreed to participate in the survey, and the participation rate was $98.1 \%$. The subjects who failed to provided information about lipid parameters (HDL-C: $n=20$; LDL-C: $n=$ 1; TG: $n=8)$ or ACR $(n=157)$ were excluded from the analyses. Finally, a total of 9730 eligible individuals were included in the data analyses. The study protocol was approved by the Institutional Review Board of the Sun Yat-sen Memorial Hospital affiliated with Sun Yat-sen University and was in accordance with the principles of the Helsinki Declaration II. Written informed consent was obtained from each participant prior to data collection.

\section{Clinical and biochemical measurements}

A standard questionnaire was administered by trained staff to collect information about sociodemographic characteristics, family history and lifestyle factors. Smoking or drinking habits were classified as 'never', 'current' (smoking or drinking regularly in the past 6 months) or 'ever' (cessation of smoking or drinking of more than 6 months) [20]. A short form of the International Physical Activity Questionnaire (IPAQ) was used to estimate physical activity during leisure time by adding the results for questions about the frequency and duration of moderate or vigorous activities and walking [21]. Metabolic equivalent hours per week (MET-h/week) were calculated separately to evaluate total physical activity.

All participants completed the anthropometrical measurements with the assistance of trained staff using standard protocols [18]. Blood pressure was measured three times consecutively by the same observer with 5 min intervals using an automated electronic device (OMRON, Omron Company, China). The average of the three measurements of blood pressure was used for the analysis. Body height and body weight were recorded to the nearest $0.1 \mathrm{~cm}$ and $0.1 \mathrm{~kg}$, respectively, while participants were wearing light indoor clothing without shoes. Body mass indices (BMI) were calculated as the weight in kilograms divided by the height in meters squared $\left(\mathrm{kg} / \mathrm{m}^{2}\right)$. Obesity was defined by BMI equal to or greater than 28 , and overweight was defined by BMI equal to or greater than 24 and less than 28 [22]. Waist circumference (WC) was measured at the umbilical level with participant in the standing position at the end of a gentle expiration. All relevant data was recorded into the database by 2 different persons to ascertain results reliability in the present study. We performed source data confirmation including verification of all relevant data and informed consent of participating participants. Data queries will be raised for inconsistent, impossible or missing data to ensure that all data are reliable and have been processed correctly.

Venous blood samples were collected for laboratory tests after an overnight fasting of at least $10 \mathrm{~h}$. Measurements of fasting serum insulin, fasting plasma glucose (FPG), oral glucose tolerance test (OGTT) $2 \mathrm{~h}$ glucose, triglycerides (TG), total cholesterol (TC), high-density lipoprotein cholesterol (HDL-C), low-density lipoprotein cholesterol (LDL-C) and serum creatinine $(\mathrm{SCr})$ were performed with an autoanalyzer (Beckman CX-7 Biochemical Autoanalyser, Brea, CA, USA). Non-HDL-C levels were calculated from the difference between serum TC and HDL-C. Dyslipidemia was determined if any one of the following indexes was met: TC 
level $\geq 240 \quad \mathrm{mg} / \mathrm{dL}, \quad$ LDL-C level $\geq 160 \mathrm{mg} / \mathrm{dL}$, TG level $\geq 200 \mathrm{mg} / \mathrm{dL}$, HDL-C level $<40 \mathrm{mg} / \mathrm{dL}$ or previously diagnosed dyslipidemia [23]. Hemoglobin A1c (HbA1c) was assessed by high-performance liquid chromatography (Bio-Rad, Hercules, CA). The insulin resistance index (homeostasis model assessment of insulin resistance, HOMA-IR) was calculated as fasting insulin $(\mu \mathrm{IU} / \mathrm{ml}) \times$ fasting glucose $(\mathrm{mmol} / \mathrm{L}) / 22.5$ [24]. Insulin resistance was defined by a HOMA-IR index within the top quartile (greater than 2.54 in the present study) [25]. Diabetes was diagnosed according to the 1999 World Health Organization diagnostic criteria. The abbreviated Modification of Diet in Renal Disease (MDRD) formula recalibrated for Chinese population was used to calculate estimated glomerular filtration rate (eGFR) expressed in $\mathrm{mL} / \mathrm{min}$ per $1.73 \mathrm{~m}^{2}$ using a formula of eGFR $=186 \times$ [serum creatinine $\times$ $0.011]^{-1.154} \times$ [age $^{-0.203} \times[0.742$ if female $] \times 1.233$, where serum creatinine was expressed as $\mu \mathrm{mol} / \mathrm{L}$ and 1.233 was the adjusting coefficient for Chinese population [26].

\section{Definition of low-grade albuminuria, increased urinary albumin excretion and chronic kidney disease}

Definitions of abnormalities in albumin excretion were according to the latest guidelines of American Diabetes Association's Standards of Medical Care [3]. The first morning spot urine samples were collected for assessing the ACR. Urine albumin and creatinine were measured by chemiluminescence immunoassay (Siemens Immulite 2000, United States) and the Jaffe' s kinetic method (Biobase-Crystal, Jinan, China) on the automatic analyzer, respectively. ACR was calculated by dividing the urinary albumin concentrations by the urinary creatinine concentrations and expressed in $\mathrm{mg} / \mathrm{g}$. Increased urinary albumin excretion was defined according to the ACR ranges greater or equal than $30 \mathrm{mg} / \mathrm{g}$. The definition of low-grade albuminuria was according to the highest quartile of ACR in participants without increased urinary albumin excretion (ACR greater or equal than $11 \mathrm{mg} / \mathrm{g}$ and less than $30 \mathrm{mg} / \mathrm{g}$ in the present study). Chronic kidney disease (CKD) was defined as eGFR less than $60 \mathrm{~mL} / \mathrm{min}$ per $1.73 \mathrm{~m}^{2}$ or presence of albuminuria (ACR greater or equal than $30 \mathrm{mg} / \mathrm{g}$ ).

\section{Statistical analyses}

The statistical analyses were performed using SAS version 9.2 (SAS Institute Inc., Cary, NC, USA). Totally, we analyzed the effects of lipid parameters (TG, TC, HDLC, LDL-C, Non-HDL-C, Non-HDL-C/HDL-C and TG/ HDL-C) on clinical factors correlated with renal function (ACR, SCr and eGFR), prevalence of albuminuria (low-grade albuminuria, increased urinary albumin excretion) and CKD.
Continuous variables are presented as the means \pm the standard deviations (SD) with the exception of skewed variables, which were presented as medians (interquartile ranges). Categorical variables are expressed as numbers (proportions). FPG, TG, ACR, HOMA-IR, Non-HDL-C/HDL-C, TG/HDL-C, SCr, eGFR and MET-h/week were logarithmically transformed prior to analysis due to non-normal distributions. Linear regression analyses were used to test for trends across groups. Differences between groups were tested with oneway ANOVAs, and post hoc comparisons were performed using the Bonferroni correction. Comparisons between categorical variables were performed with the $\chi^{2}$ test. Pearson's correlation and multivariate linear regression model were performed to evaluate the associations of lipid parameters with ACR, SCr and eGFR. Unadjusted and multivariate adjusted logistic regression analyses were used to assess the prevalence of low-grade albuminuria, increased urinary albumin excretion and CKD according to elevated lipid profiles quartiles. Participants with CKD were excluded from the analysis for risk of prevalent low-grade albuminuria. The odds ratios (OR) and the corresponding $95 \%$ confidence intervals (95\% CI) were calculated. Model 1 was unadjusted. Model 2 was adjusted for age and sex. Model 3 is further adjusted for BMI, current smoking and drinking status, physical activity level, systolic blood pressure (SBP), and HbA1c. Model 4 is further adjusted for previously diagnosed diabetes, cardiovascular diseases, hypertension and dyslipidemia. The relationships of lipid parameters with increased urinary albumin excretion and CKD were also explored within subgroups that stratified by age $(\geq 55$ or $<55$ years), degree of obesity (normal, overweight or obesity), hypertension (yes or no), diabetes (yes or no) and dyslipidemia (yes or no). In interaction analyses, we examined separately for feasible associate factors that could modify the relationship between albuminuria and lipid measures. Tests for interaction were performed with including simultaneously each strata factor, lipid parameters quartiles and the respective interaction terms (strata factor multiplied by lipid parameters quartiles) in the final model.

All statistical tests were two-sided, and $P$ values $<0.05$ were considered statistically significant.

\section{Results}

Basic clinical characteristics of the study population

Among the 9730 enrolled subjects, the mean age was $55.9 \pm 8.1$ years and median ACR was $8.1 \mathrm{mg} / \mathrm{g}$ with interquartile range 5.7 to $12.2 \mathrm{mg} / \mathrm{g}$. There were 2274 (23.4 \%) participants categorized as low-grade albuminuria, 639 (6.6 \%) participants categorized as increased urinary albumin excretion and 689 (7.1\%) participants categorized as CKD, respectively. The clinical and biochemical 
characteristics according to urinary albumin excretion status were presented in Table 1. Compared subjects with normal urinary albumin excretion, those with low-grade albuminuria or increased urinary albumin excretion had significantly higher TG, Non-HDL-C, Non-HDL-C/HDL-C and TG/HDL-C (all $P<0.05$ ). Moreover, compared subjects with low-grade albuminuria, those with increased urinary albumin excretion presented with higher TG, Non-HDL-C/ HDL-C, TG/HDL-C and lower HDL-C level (all $P<0.05$ ).

\section{Associations between lipid parameters and clinical factors correlated with renal function}

As shown in Table 2, Pearson's correlation analysis revealed that TG, HDL-C, Non-HDL-C/HDL-C, TG/ HDL-C were significantly correlated with ACR, SCr and
eGFR (all $P<0.0001$ ). By performing multivariate linear regression analysis and further adjusted for age and sex, we found that the associations were still persisted (all $P<0.0001)$. However, TC and LDL-C were not correlated with ACR in both Pearson's correlation and linear regression analysis.

\section{Associations between lipid parameters and low-grade albuminuria, increased urinary albumin excretion and CKD}

Figure 1 showed the prevalent low-grade albuminuria, increased urinary albumin excretion and CKD in different lipid parameters quartiles. The prevalence of albuminuria and CKD were tended to increase with the elevated TG, Non-HDL-C, Non-HDL-C/HDL-C and

Table 1 Characteristics of study population by urinary albumin excretion status

\begin{tabular}{|c|c|c|c|c|}
\hline & Normal urinary albumin excretion & Low-grade albuminuria & Increased urinary albumin excretion & $P$ for trend \\
\hline$n(\%)$ & $6817(70.1)$ & $2274(23.4)$ & $639(6.6)$ & \\
\hline ACR (mg/g) & $6.7(5.0-8.4)$ & $14.7(12.5-19.0)^{*}$ & $53.3(37.8-94.6)^{* \#}$ & $<0.0001$ \\
\hline Age (years) & $55.5 \pm 7.7$ & $56.7 \pm 8.4^{*}$ & $58.0 \pm 9.6^{* \#}$ & $<0.0001$ \\
\hline Male $[\mathrm{n}(\%)]$ & 2097 (30.8) & $501(22.0)$ & $188(29.4)$ & $<0.0001$ \\
\hline $\mathrm{BMI}\left(\mathrm{kg} / \mathrm{m}^{2}\right)$ & $23.4 \pm 3.2$ & $24.0 \pm 3.8^{*}$ & $24.7 \pm 3.7^{* \#}$ & $<0.0001$ \\
\hline$W C(\mathrm{~cm})$ & $81.1 \pm 9.2$ & $82.3 \pm 9.9^{*}$ & $85.1 \pm 10.4^{* \#}$ & $<0.0001$ \\
\hline $\mathrm{SBP}(\mathrm{mmHg})$ & $124.2 \pm 15.6$ & $129.0 \pm 16.9^{*}$ & $135.0 \pm 18.9^{* \#}$ & $<0.0001$ \\
\hline $\mathrm{DBP}(\mathrm{mmHg})$ & $74.6 \pm 9.5$ & $76.4 \pm 10.4^{*}$ & $78.9 \pm 11.0^{*}$ & $<0.0001$ \\
\hline Current smoker $[n(\%)]$ & $703(10.6)$ & $164(7.4)^{*}$ & $74(11.8)^{\#}$ & 0.072 \\
\hline Current drinker [n (\%)] & $226(3.4)$ & $67(3.1)$ & $23(3.7)$ & 0.817 \\
\hline Physical activity (MET-h/week) & $21.0(10.5-45.0)$ & $21.0(10.5-42.0)$ & $21.5(10.5-42.0)$ & 0.831 \\
\hline $\mathrm{TG}(\mathrm{mg} / \mathrm{dL})$ & $108.8(79.6-157.5)$ & $115.9(82.3-169.9)^{*}$ & $137.2(96.5-194.7)^{* \#}$ & $<0.0001$ \\
\hline $\mathrm{TC}(\mathrm{mg} / \mathrm{dL})$ & $199.6 \pm 47.4$ & $203.6 \pm 48.7^{*}$ & $202.4 \pm 52.0$ & 0.002 \\
\hline $\mathrm{HDL}-\mathrm{C}(\mathrm{mg} / \mathrm{dL})$ & $51.2 \pm 13.9$ & $51.1 \pm 13.8$ & $47.8 \pm 12.8^{* \#}$ & $<0.0001$ \\
\hline $\mathrm{LDL}-\mathrm{C}(\mathrm{mg} / \mathrm{dL})$ & $120.8 \pm 36.5$ & $122.2 \pm 37.8$ & $122.6 \pm 39.0$ & 0.067 \\
\hline Non-HDL-C (mg/dl) & $148.4 \pm 41.8$ & $152.4 \pm 43.2^{*}$ & $154.6 \pm 46.3^{*}$ & $<0.0001$ \\
\hline Non-HDL-C/HDL-C & $2.9(2.3-3.6)$ & $3.0(2.4-3.8)^{*}$ & $3.3(2.6-4.0)^{* \#}$ & $<0.0001$ \\
\hline TG/HDL-C & $2.2(1.5-3.5)$ & $2.3(1.5-3.7)^{*}$ & $3.0(1.9-4.5)^{* \#}$ & $<0.0001$ \\
\hline $\mathrm{FPG}(\mathrm{mmol} / \mathrm{L})$ & $5.4(5.0-5.9)$ & $5.5(5.0-6.1)^{*}$ & $5.6(5.1-6.7)^{* \#}$ & $<0.0001$ \\
\hline $\mathrm{HbA} 1 \mathrm{C}(\%)$ & $6.0 \pm 0.7$ & $6.2 \pm 1.0^{*}$ & $6.5 \pm 1.5^{* \#}$ & $<0.0001$ \\
\hline HOMA-IR & $1.67(1.19-2.39)$ & $1.88(1.29-2.80)^{*}$ & $2.23(1.45-3.48)^{* \#}$ & $<0.0001$ \\
\hline $\mathrm{SCr}(\mu \mathrm{mol} / \mathrm{L})$ & $67.0(60.8-76.0)$ & $65.0(59.5-72.9)^{*}$ & $69.1(60.9-81.1)^{* \#}$ & 0.531 \\
\hline eGFR $\left(\mathrm{ml} / \mathrm{min}\right.$ per $\left.1.73 \mathrm{~m}^{2}\right)$ & $110.9(100.0-123.8)$ & $111.5(100.1-124.8)$ & $106.4(94.2-122.0)^{* \#}$ & $<0.0001$ \\
\hline Previous CVD (\%) & $205(3.0)$ & $78(3.4)$ & $35(5.5)^{* \#}$ & 0.003 \\
\hline Previous hypertension (\%) & $893(13.1)$ & $486(21.4)^{*}$ & $198(31.0)^{* \#}$ & $<0.0001$ \\
\hline Previous diabetes (\%) & $313(4.6)$ & $201(8.8)^{*}$ & $90(14.1)^{* \#}$ & $<0.0001$ \\
\hline Previous dyslipidemia (\%) & $325(4.8)$ & $124(5.5)$ & $49(7.7)^{* \#}$ & 0.002 \\
\hline
\end{tabular}

1. Data were means \pm SD or medians (interquartile ranges) for skewed variables or numbers (proportions) for categorical variables.

2. $P$ for trend was calculated for the linear regression analysis tests across the groups. $P$ values were for the ANOVA or $X 2$ analyses across the groups.

3. ${ }^{*} \mathrm{P}<0.05$ compared with normal urinary albumin excretion group; ${ }^{\#} \mathrm{P}<0.05$ compared with low-grade albuminuria group.

4. ACR, urinary albumin to creatinine ratio; BMI, body mass index; WC, waist circumference; SBP, systolic blood pressure; DBP, diastolic blood pressure; TG, triglycerides; TC, total cholesterol; HDL-C, high-density lipoprotein cholesterol; LDL-C, low-density lipoprotein cholesterol; FPG, fasting plasma glucose; HOMA-IR, homeostasis model assessment of insulin resistance; $\mathrm{SCr}$, serum creatinine; eGFR, estimated glomerular filtration rate; CVD, cardiovascular diseases. 
Table 2 Pearson's correlation and multiple regression analysis of lipid parameters associated with ACR, SCr and eGFR

\begin{tabular}{|c|c|c|c|c|c|c|c|c|c|c|c|c|}
\hline & \multicolumn{4}{|c|}{ ACR $(\mathrm{mg} / \mathrm{g})$} & \multicolumn{4}{|c|}{$\mathrm{SCr}(\mu \mathrm{mol} / \mathrm{L})$} & \multicolumn{4}{|c|}{ eGFR $\left(\mathrm{ml} / \mathrm{min}\right.$ per $\left.1.73 \mathrm{~m}^{2}\right)$} \\
\hline & $r$ & $P$ value & Standardized $\beta$ & $P$ value & $r$ & $P$ value & Standardized $\beta$ & $P$ value & $r$ & $P$ value & Standardized $\beta$ & $P$ value \\
\hline $\mathrm{TG}(\mathrm{mg} / \mathrm{dl})$ & 0.09 & $<0.0001$ & 0.09 & $<0.0001$ & 0.23 & $<0.0001$ & 0.19 & $<0.0001$ & -0.23 & $<0.0001$ & -0.22 & $<0.0001$ \\
\hline $\mathrm{TC}(\mathrm{mg} / \mathrm{dl})$ & 0.01 & 0.177 & -0.01 & 0.556 & 0.25 & $<0.0001$ & 0.29 & $<0.0001$ & -0.37 & $<0.0001$ & -0.33 & $<0.0001$ \\
\hline $\mathrm{HDL}-\mathrm{C}(\mathrm{mg} / \mathrm{dl})$ & -0.05 & $<0.0001$ & -0.07 & $<0.0001$ & 0.05 & $<0.0001$ & 0.19 & $<0.0001$ & -0.21 & $<0.0001$ & -0.23 & $<0.0001$ \\
\hline LDL-C (mg/dl) & 0.002 & 0.846 & -0.01 & 0.172 & 0.20 & $<0.0001$ & 0.22 & $<0.0001$ & -0.29 & $<0.0001$ & -0.26 & $<0.0001$ \\
\hline $\begin{array}{l}\text { Non-HDL-C } \\
(\mathrm{mg} / \mathrm{dl})\end{array}$ & 0.03 & 0.002 & 0.02 & 0.123 & 0.27 & $<0.0001$ & 0.26 & $<0.0001$ & -0.32 & $<0.0001$ & -0.31 & $<0.0001$ \\
\hline $\begin{array}{l}\text { Non-HDL-C/ } \\
\text { HDL-C }\end{array}$ & 0.06 & $<0.0001$ & 0.07 & $<0.0001$ & 0.20 & $<0.0001$ & 0.08 & $<0.0001$ & -0.13 & $<0.0001$ & -0.10 & $<0.0001$ \\
\hline $\mathrm{TG} / \mathrm{HDL}-\mathrm{C}$ & 0.09 & $<0.0001$ & 0.10 & $<0.0001$ & 0.16 & $<0.0001$ & 0.06 & $<0.0001$ & -0.09 & $<0.0001$ & -0.06 & $<0.0001$ \\
\hline
\end{tabular}

1. ACR, urinary albumin to creatinine ratio; TG, triglycerides; TC, total cholesterol; HDL-C, high-density lipoprotein cholesterol; LDL-C, low-density lipoprotein

cholesterol; $\mathrm{SCr}$, serum creatinine; eGFR, estimated glomerular filtration rate.

2. ACR, SCr, eGFR, TG, Non-HDL-C/HDL-C and TG/HDL-C levels were logarithmically transformed to achieve a normal distribution.

3. $r$, correlation coefficient; $\beta$, regression coefficient; Multiple regression analysis is adjusted for age and sex.

TG/HDL-C quartiles (all P for trend $<0.001$ ). For both prevalent increased urinary albumin excretion and CKD, no obvious trend differences were detected in subjects with different quartiles of TC and LDL-C. The prevalence of low-grade albuminuria tended to increase with lipid parameters quartiles except for HDL-C and LDL-C.

As shown in Table 3, in the univariable logistic regression analysis, participants were more likely to have lowgrade albuminuria with the elevated quartiles of TG, TC, Non-HDL-C, Non-HDL-C/HDL-C and TG/HDL-C. However, in the multivariable logistic regression, no significant relationships between those lipid parameters and low-grade albuminuria were observed. Similarly in Table 3, in the univariable logistic regression analysis, with the quartile change of HDL-C, Non HDL-C, NonHDL-C/HDL-C and TG/HDL-C, participants were more likely to have prevalent increased urinary albumin excretion and CKD. Further adjustments for other potential confounding factors including age, sex, BMI, current smoking and drinking status, physical activity level, SBP, HbA1c, previously diagnosed diabetes, cardiovascular diseases, hypertension and dyslipidemia, the associations of those lipid measures with increased urinary albumin excretion and CKD were still persisted.

Compare with other lipid parameters, TG/HDL-C have shown the strongest correlation with increased odds of both increased urinary albumin excretion and CKD across all logistic regression models (Table 3). To verify the stability of such results, we conducted stratified analyses to determine the odds of increased urinary albumin excretion and CKD with each quartile increase of TG/HDL-C in different subgroups. As shown in Figs. 2 and 3, such associations were not consistently the same according to stratified factors. The associations of TG/HDL-C with prevalent increased urinary albumin excretion and CKD were significant in women strata, both age strata $(\geq 55$ and $<55$ ), BMI strata (normal and overweight), both hypertension strata (yes and no), both diabetes strata (yes and no) and subjects without dyslipidemia strata. The difference in the subgroups analysis was accompanied by a statistically significant interaction term when stratified by sex $(P=0.021$ for increased urinary albumin excretion and $P=0.010$ for CKD).

\section{Discussion}

In this study of 9730 middle-aged and elderly Chinese, we found significant discordant associations of lipid parameters with albuminuria and CKD. TG/HDL-C is a better marker than other routine lipid measures for identifying both increased urinary albumin excretion and CKD, which should be given more consideration in the clinical approach to risk reduction among those patients. Our data do not support the association between routinely available lipid measures and prevalent low-grade albuminuria.

Lipoprotein abnormalities have been identified as possible causes of renal function damage. Previous study has shown that elevated TG levels and diminished HDL$C$ levels, but not LDL-C, were associated with increased risk of renal dysfunction and poor renal outcomes [27]. Significant associations between the TG/HDL-C and reduced eGFR or albuminuria have also been identified recently in Korean and Japanese adults [28-30]. In addition to the above lipid measures, a recent study also found that Non-HDL-C/HDL-C is an independent risk factor for the development of CKD [31]. Systemically clarified correlations of different lipid measures with albuminuria and CKD would probably shed light on the prevention and treatment of the diseases. Additionally, growing evidence indicated that low-grade albuminuria is associated with impaired kidney function and incident cardiovascular diseases [32, 33]. Less is known concerning the association between lipid profiles and low-grade 


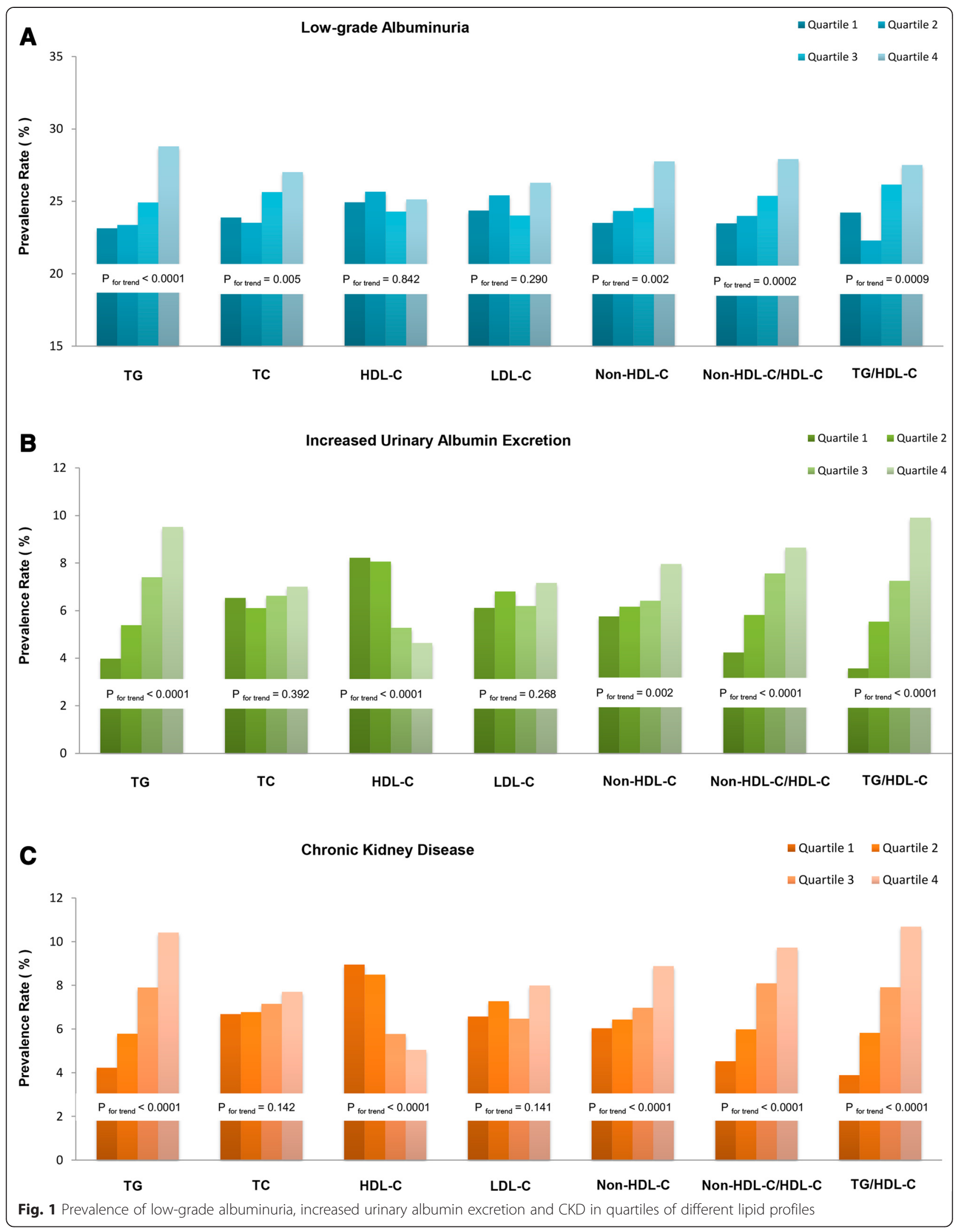


Table 3 Association between lipid parameters and prevalent low-grade albuminuria, increased urinary albumin excretion and CKD

\begin{tabular}{|c|c|c|c|c|c|}
\hline & & \multicolumn{4}{|c|}{ 1-Quartile change of lipid parameters ${ }^{a}$} \\
\hline & & Model 1 & Model 2 & Model 3 & Model 4 \\
\hline \multirow[t]{7}{*}{ Low-grade albuminuria } & TG & $1.10(1.06-1.15)$ & $1.10(1.06-1.15)$ & $1.01(0.96-1.06)$ & $1.01(0.97-1.06)$ \\
\hline & $\mathrm{TC}$ & $1.06(1.02-1.11)$ & $1.03(0.98-1.07)$ & $1.01(0.96-1.05)$ & $1.02(0.97-1.06)$ \\
\hline & $\mathrm{HDL}-\mathrm{C}$ & $1.00(0.96-1.05)$ & $1.05(1.01-1.10)$ & $0.99(0.95-1.04)$ & $0.99(0.94-1.04)$ \\
\hline & LDL-C & $1.02(0.98-1.07)$ & $0.99(0.95-1.04)$ & $0.97(0.93-1.02)$ & $0.98(0.94-1.03)$ \\
\hline & Non-HDL-C & $1.07(1.03-1.12)$ & $1.05(1.00-1.09)$ & $1.00(0.96-1.05)$ & $1.01(0.96-1.06)$ \\
\hline & Non-HDL-C/HDL-C & $1.08(1.04-1.13)$ & $1.10(1.05-1.15)$ & $1.01(0.96-1.06)$ & $1.01(0.96-1.07)$ \\
\hline & $\mathrm{TG} / \mathrm{HDL}-\mathrm{C}$ & $1.08(1.03-1.12)$ & $1.09(1.05-1.14)$ & $0.99(0.94-1.04)$ & $0.99(0.94-1.04)$ \\
\hline \multirow[t]{7}{*}{ Increased urinary albumin excretion } & TG & $1.36(1.27-1.47)$ & $1.34(1.24-1.44)$ & $1.16(1.07-1.26)$ & $1.17(1.08-1.27)$ \\
\hline & $\mathrm{TC}$ & $1.03(0.96-1.11)$ & $1.01(0.94-1.08)$ & $0.95(0.88-1.02)$ & $0.96(0.89-1.04)$ \\
\hline & $\mathrm{HDL}-\mathrm{C}$ & $1.25(1.16-1.35)$ & $1.26(1.17-1.36)$ & $1.17(1.08-1.27)$ & $1.16(1.07-1.26)$ \\
\hline & LDL-C & $1.04(0.97-1.12)$ & $1.02(0.95-1.09)$ & $0.96(0.89-1.04)$ & $0.98(0.91-1.06)$ \\
\hline & Non-HDL-C & $1.12(1.04-1.20)$ & $1.09(1.01-1.17)$ & $0.99(0.91-1.07)$ & $1.00(0.93-1.08)$ \\
\hline & Non-HDL-C/HDL-C & $1.28(1.19-1.38)$ & $1.25(1.16-1.35)$ & $1.09(1.00-1.18)$ & $1.10(1.01-1.19)$ \\
\hline & TG/HDL-C & $1.42(1.32-1.53)$ & $1.40(1.30-1.51)$ & $1.21(1.11-1.31)$ & $1.21(1.11-1.31)$ \\
\hline \multirow[t]{7}{*}{ CKD } & $\mathrm{TG}$ & $1.38(1.29-1.48)$ & $1.35(1.25-1.45)$ & $1.18(1.09-1.28)$ & $1.19(1.10-1.28)$ \\
\hline & $\mathrm{TC}$ & $1.05(0.98-1.13)$ & $1.03(0.96-1.10)$ & $0.97(0.90-1.05)$ & $0.99(0.92-1.07)$ \\
\hline & $\mathrm{HDL}-\mathrm{C}$ & $1.25(1.16-1.34)$ & $1.25(1.16-1.34)$ & $1.16(1.07-1.25)$ & $1.15(1.06-1.24)$ \\
\hline & LDL-C & $1.05(0.98-1.13)$ & $1.03(0.96-1.10)$ & $0.98(0.91-1.05)$ & $0.99(0.92-1.07)$ \\
\hline & Non-HDL-C & $1.15(1.07-1.23)$ & $1.11(1.04-1.20)$ & $1.02(0.94-1.10)$ & $1.03(0.96-1.11)$ \\
\hline & Non-HDL-C/HDL-C & $1.32(1.23-1.41)$ & $1.28(1.19-1.37)$ & $1.11(1.03-1.20)$ & $1.12(1.04-1.22)$ \\
\hline & $\mathrm{TG} / \mathrm{HDL}-\mathrm{C}$ & $1.42(1.32-1.53)$ & $1.39(1.30-1.50)$ & $1.21(1.11-1.31)$ & $1.21(1.11-1.31)$ \\
\hline
\end{tabular}

Data are odds ratios (95\% confidence interval). Participants without low-grade albuminuria, increased urinary albumin excretion or CKD are defined as 0 and with low-grade albuminuria, increased urinary albumin excretion or CKD as 1

Model 1 is unadjusted

Model 2 is adjusted for age and sex

Model 3 is further adjusted for BMl, current smoking and drinking status, physical activity level, SBP and HbA1c

Model 4 is further adjusted for previously diagnosed diabetes, cardiovascular diseases, hypertension and dyslipidemia

${ }^{a}$ All variables were calculated for 1-Quartile increasing of lipid parameters except for HDL-C, which was calculated for 1-Quartile decreasing

albuminuria. In this study, compared with other lipid measures, TG/HDL-C has shown the most significant association with increased urinary albumin excretion and CKD after adjustment for demographic and clinical information. However, no association of lipid measures with low-grade albuminuria was detected after adjustment for same variables.

There are several reasons why the level of TG/HDL-C may be superior to that of other lipid parameters in increased urinary albumin excretion and CKD identification. Elevated TG levels and decreased HDL-C levels have been most strongly associated with increased risk of renal dysfunction [27, 34]. Despite divergent underlying mechanisms, injury-induced cellular TG accumulation can disrupt and damage cellular homeostasis. According to the severity of the injury, TG accumulation could result in renal tubular cell damage in vivo and in vitro models [35]. As a medium of interaction with cell surface proteins, HDL-C could promote TC clearance from the circulation and decrease lipid deposition in the arterial wall, which might slow the progression of kidney dysfunction in patients with CKD $[34,36]$. The nature of TG to HDL-C ratio seems to be better correlated with the development of CKD and albuminuria than single measurement of either TG or HDL-C levels. Moreover, insulin resistance is associated with albuminuria and the development of CKD in observational human studies [37]. Compared with other metabolic markers, TG/ HDL-C in circulation is better correlated with insulin resistance, which might therefore relevant with risk of various adverse outcomes including renal dysfunction [38]. Based on the results of the present study, the association of TG/HDL-C with increased urinary albumin excretion and CKD was stronger in participants without dyslipidemia. It is thus possible that TG/HDL-C could represent the progression of renal insufficiency even in the early stage of lipid metabolism abnormity.

Several limitations in this study require consideration. First, we evaluated the urinary albumin excretion on a spot morning urine sample. We admitted that multiple 


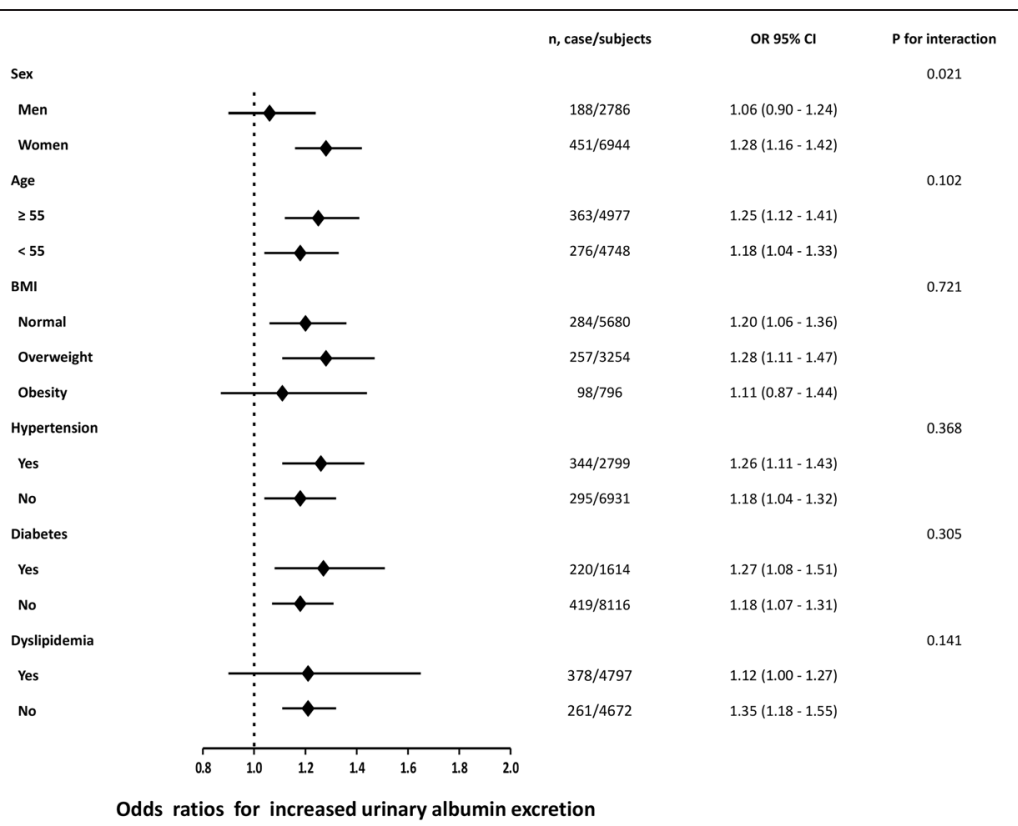

Fig. 2 Risk of prevalent increased urinary albumin excretion with each quartile increase of TG/HDL-C level in different subgroups

samples would provide more stable results for albumin excretion [39]. However, results of spot urine samples correlate well with those of $24 \mathrm{~h}$ or multiple urine samples [40, 41]. Use of spot samples for assessing urinary ACR is therefore recommended as a reliable alternative to perform in the out-patient clinic and large epidemiological specimen collection. Second, although it is possible that lipid measures is related with different degrees of albuminuria after controlled for extensive confounding factors, other unmeasured confounders, such as cystatin-C, should be also considered to evaluate to strength the findings of the present study. Third, growing evidence has suggested that abnormal lipid metabolism could contribute to the deterioration of renal function [27, 42]. However, due to the cross-sectional design of the present study, we should be cautious regarding the interpretation of whether dyslipidemia is a causal factor of or a consequence of albuminuria and CKD. Fourth, medications with angiotensin-converting enzyme inhibitors or angiotensin receptor blockers should have affected urinary

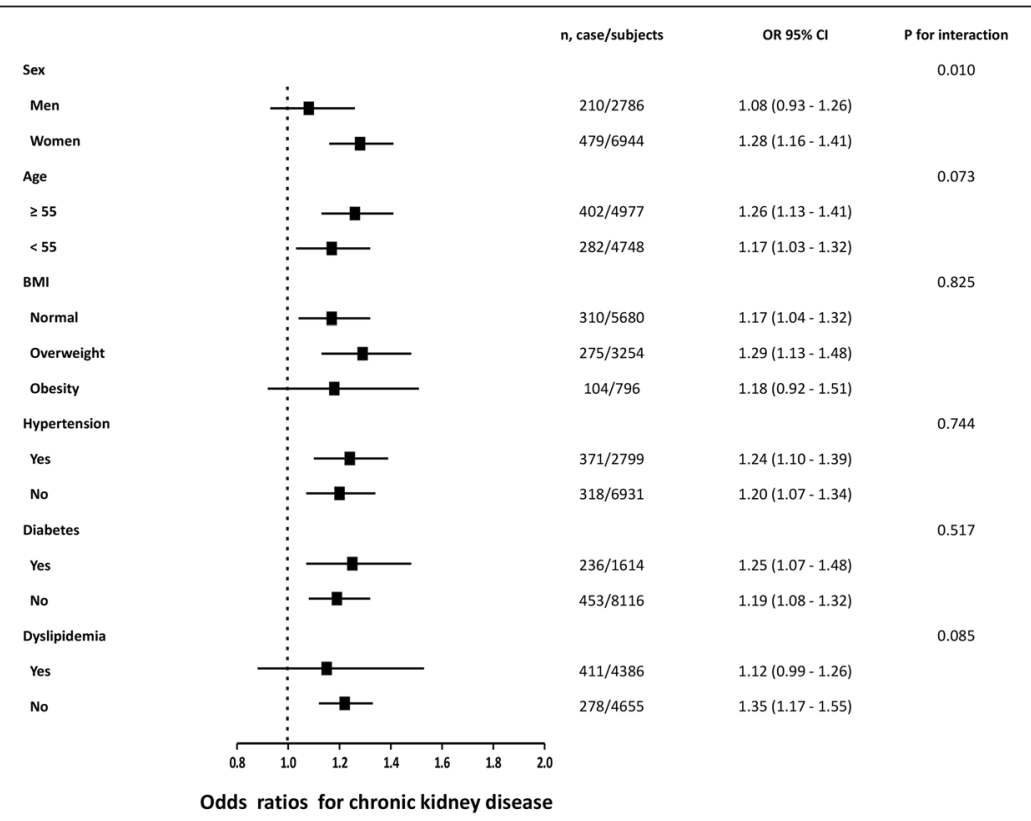

Fig. 3 Risk of prevalent CKD with each quartile increase of TG/HDL-C level in different subgroups 
albumin excretion and should be taken into account when analyzing possible risk factors associated to proteinuria. Absence of these data may influence risk estimates and result interpreting in this setting. Fifth, the results from the present study of Chinese population might not be representative of other races and younger people. The study population was predominantly female, partially because we invited residents with the mean age of 56 years and females are predominant in this age range in China.

\section{Conclusion}

In conclusion, the present study demonstrates discordant associations of lipid parameters with renal insufficiency and TG/HDL-C is a better marker for evaluating increased urinary albumin excretion and CKD. No statistically significant association between lipid parameters and prevalent low-grade albuminuria was found in our data.

\section{Ethics, consent and permissions}

The study protocol was approved by the Institutional Review Board of the Sun Yat-sen Memorial Hospital affiliated with Sun Yat-sen University and was in accordance with the principles of the Helsinki Declaration II. Written informed consent was obtained from each participant prior to data collection.

\section{Consent to publish}

We have obtained consent to publish from the participant to report individual patient data.

\begin{abstract}
Abbreviations
ACR: Urinary albumin to creatinine ratio; BMl: Body-mass index; Cl: Confidence intervals; CVD: Cardiovascular diseases; DBP: Diastolic blood pressure; eGFR: Estimated glomerular filtration rate; FPG: Fasting plasma glucose; HbA1c: Hemoglobin A1c; TG: Triglycerides; TC: total cholesterol; HDL-C: High-density lipoprotein cholesterol; LDL-C: Low-density lipoprotein cholesterol; HOMA-IR: Homeostasis model assessment of insulin resistance; MET-h/week: Metabolic equivalent hours per week; OR: Odds ratios; SBP: Systolic blood pressure; SCr: Serum creatinine; SD: Standard deviation; WC: Waist circumference.
\end{abstract}

\section{Competing interests}

All of the authors declare that they have no relevant conflicts of interests.

\section{Authors' contributions}

Conceived and designed the experiments: $L Y, M R$ and $Y L$; performed the experiments: DL, CH, FL, YQ, JT and SX; analyzed the data: KS; YC and DL; wrote the manuscript: $\mathrm{KS}$ and $\mathrm{DL}$. All authors read and approved the final manuscript.

\section{Acknowledgments}

We are indebted to the participants of the present study for their persistent and outstanding support and to our colleagues for their valuable assistance.

\section{Funding}

This work was supported by grants from the following sources: 1) the National Natural Science Foundation of China (81471034,81370910); 2) Major project of the people's livelihood science and technology in Guangzhou (201300000102); 3) The Natural Science Foundation of Guangdong Province (S2013010016443); 4) The 5010 Clinical Medical Project of Sun Yat-sen University (No.2010010); 5) Grants from the Chinese Society of Endocrinology and the National Clinical Research Center for Metabolic Diseases.

\section{Statement of authorship}

All authors believe that this manuscript represents valid work and have reviewed and approved the final version. The work has not been published previously and is not under consideration for publication elsewhere in part or in whole.

Received: 29 August 2015 Accepted: 6 November 2015

Published online: 25 November 2015

\section{References}

1. Go AS, Chertow GM, Fan D, McCulloch CE, Hsu CY. Chronic kidney disease and the risks of death, cardiovascular events, and hospitalization. N Engl J Med. 2004;351(13):1296-305.

2. Zhang L, Wang F, Wang L, Wang W, Liu B, Liu J, et al. Prevalence of chronic kidney disease in China: a cross-sectional survey. Lancet. 2012;379(9818): 815-22.

3. American Diabetes Association. Standards of medical care in diabetes-2014. Diabetes Care. 2014;37 Suppl 1:S14-80.

4. de Zeeuw D, Remuzzi G, Parving HH, Keane WF, Zhang Z, Shahinfar S, et al. Albuminuria, a therapeutic target for cardiovascular protection in type 2 diabetic patients with nephropathy. Circulation. 2004;110(8):921-7.

5. Deckert T, Feldt-Rasmussen B, Borch-Johnsen $\mathrm{K}$, Jensen T, KofoedEnevoldsen A. Albuminuria reflects widespread vascular damage. The Steno hypothesis. Diabetologia. 1989;32(4):219-26.

6. Gerstein HC, Mann JF, Yi Q, Zinman B, Dinneen SF, Hoogwerf B, et al. Albuminuria and risk of cardiovascular events, death, and heart failure in diabetic and nondiabetic individuals. JAMA. 2001;286(4):421-6.

7. Katz DH, Selvaraj S, Aguilar FG, Martinez EE, Beussink L, Kim KY, et al. Association of low-grade albuminuria with adverse cardiac mechanics: findings from the hypertension genetic epidemiology network (HyperGEN) study. Circulation. 2014;129(1):42-50.

8. Arnlov J, Evans JC, Meigs JB, Wang TJ, Fox CS, Levy D, et al. Low-grade albuminuria and incidence of cardiovascular disease events in nonhypertensive and nondiabetic individuals: the Framingham Heart Study. Circulation. 2005;112(7):969-75.

9. Ingelsson E, Sundstrom J, Lind L, Riserus U, Larsson A, Basu S, et al. Lowgrade albuminuria and the incidence of heart failure in a community-based cohort of elderly men. Eur Heart J. 2007;28(14):1739-45.

10. Lloyd-Jones DM, Hong Y, Labarthe D, Mozaffarian D, Appel L, Van Horn L, et al. Defining and setting national goals for cardiovascular health promotion and disease reduction: the American Heart Association's strategic Impact Goal through 2020 and beyond. Circulation. 2010;121(4): 586-613.

11. Upadhyay A, Earley A, Lamont JL, Haynes S, Wanner C, Balk EM. Lipidlowering therapy in persons with chronic kidney disease: a systematic review and meta-analysis. Ann Intern Med. 2012;157(4):251-62.

12. de Boer $I H$, Astor BC, Kramer H, Palmas W, Seliger SL, Shlipak MG, et al. Lipoprotein abnormalities associated with mild impairment of kidney function in the multi-ethnic study of atherosclerosis. Clin J Am Soc Nephrol. 2008;3(1):125-32.

13. Kalra PA, Guo H, Kausz AT, Gilbertson DT, Liu J, Chen SC, et al. Atherosclerotic renovascular disease in United States patients aged 67 years or older: risk factors, revascularization, and prognosis. Kidney Int. 2005;68(1):293-301.

14. Global Burden of Metabolic Risk Factors for Chronic Diseases Collaboration. Cardiovascular disease, chronic kidney disease, and diabetes mortality burden of cardiometabolic risk factors from 1980 to 2010: a comparative risk assessment. Lancet Diabetes Endocrinol. 2014; 2(8):634-47.

15. Bikbov B, Perico N, Remuzzi G. High serum cholesterol: a missed risk factor for chronic kidney disease mortality. Lancet Diabetes Endocrinol. 2014;2(8):613-4.

16. Boekholdt SM, Arsenault BJ, Mora S, Pedersen TR, LaRosa JC, Nestel PJ, et al. Association of LDL cholesterol, non-HDL cholesterol, and apolipoprotein B levels with risk of cardiovascular events among patients treated with statins: a meta-analysis. JAMA. 2012;307(12):1302-9.

17. Holman RR, Coleman RL, Shine BS, Stevens RJ. Non-HDL cholesterol is less informative than the total-to-HDL cholesterol ratio in predicting cardiovascular risk in type 2 diabetes. Diabetes Care. 2005;28(7):1796-7.

18. Bi Y, Lu J, Wang W, Mu Y, Zhao J, Liu C, et al. Cohort profile: risk evaluation of cancers in Chinese diabetic individuals: a longitudinal (REACTION) study. J Diabetes. 2014;6(2):147-57. 
19. Ning G. Risk Evaluation of cAncers in Chinese diabeTic Individuals: a IONgitudinal (REACTION) study. J Diabetes. 2012;4(2):172-3.

20. Sun K, Liu J, Ning G. Active smoking and risk of metabolic syndrome: a meta-analysis of prospective studies. PLoS One. 2012;7(10):e47791.

21. Tomioka K, Iwamoto J, Saeki K, Okamoto N. Reliability and validity of the International Physical Activity Questionnaire (IPAQ) in elderly adults: the Fujiwara-kyo Study. J Epidemiol. 2011;21(6):459-65.

22. Zhou BF. Effect of body mass index on all-cause mortality and incidence of cardiovascular diseases-report for meta-analysis of prospective studies open optimal cut-off points of body mass index in Chinese adults. Biomed Environ Sci. 2002;15(3):245-52.

23. Expert Panel on Detection, Evaluation, and Treatment of High Blood Cholesterol in Adults. Executive Summary of The Third Report of The National Cholesterol Education Program (NCEP) Expert Panel on Detection, Evaluation, And Treatment of High Blood Cholesterol In Adults (Adult Treatment Panel III). JAMA. 2001;285(19):2486-97.

24. Levy JC, Matthews DR, Hermans MP. Correct homeostasis model assessment (HOMA) evaluation uses the computer program. Diabetes Care. 1998;21(12): 2191-2.

25. Matthews DR, Hosker JP, Rudenski AS, Naylor BA, Treacher DF, Turner RC. Homeostasis model assessment: insulin resistance and beta-cell function from fasting plasma glucose and insulin concentrations in man. Diabetologia. 1985;28(7):412-9.

26. Ma YC, Zuo L, Chen JH, Luo Q, Yu XQ, Li Y, et al. Modified glomerular filtration rate estimating equation for Chinese patients with chronic kidney disease. J Am Soc Nephrol. 2006;17(10):2937-44.

27. Muntner P, Coresh J, Smith JC, Eckfeldt J, Klag MJ. Plasma lipids and risk of developing renal dysfunction: the atherosclerosis risk in communities study. Kidney Int. 2000;58(1):293-301.

28. Kang HT, Kim JK, Kim JY, Linton JA, Yoon JH, Koh SB. Independent association of TG/HDL-C with urinary albumin excretion in normotensive subjects in a rural Korean population. Clin Chim Acta. 2012;413(1-2):319-24.

29. Tsuruya K, Yoshida H, Nagata M, Kitazono T, Hirakata H, Iseki K, et al. Association of the triglycerides to high-density lipoprotein cholesterol ratio with the risk of chronic kidney disease: analysis in a large Japanese population. Atherosclerosis. 2014;233(1):260-7.

30. Kang HT, Shim JY, Lee YJ, Lee JE, Linton JA, Kim JK, et al. Association between the ratio of triglycerides to high-density lipoprotein cholesterol and chronic kidney disease in Korean adults: the 2005 Korean National Health and Nutrition Examination Survey. Kidney Blood Press Res. 2011; 34(3):173-9.

31. Zuo PY, Chen XL, Liu YW, Zhang R, He XX, Liu CY. Non-HDL-cholesterol to $\mathrm{HDL}$-cholesterol ratio as an independent risk factor for the development of chronic kidney disease. Nutr Metab Cardiovasc Dis. 2015;25(6):582-7.

32. Heo NJ, Ahn JM, Lee TW, Chin HJ, Na KY, Chae DW, et al. Very low-grade albuminuria reflects susceptibility to chronic kidney disease in combination with cardiovascular risk factors. Hypertens Res. 2010;33(6):573-8.

33. Klausen $\mathrm{K}$, Borch-Johnsen $\mathrm{K}$, Feldt-Rasmussen B, Jensen $\mathrm{G}$, Clausen $\mathrm{P}$, Scharling $H$, et al. Very low levels of microalbuminuria are associated with increased risk of coronary heart disease and death independently of renal function, hypertension, and diabetes. Circulation. 2004;110(1):32-5.

34. Baragetti A, Norata GD, Sarcina C, Rastelli F, Grigore L, Garlaschelli K, et al. High density lipoprotein cholesterol levels are an independent predictor of the progression of chronic kidney disease. J Intern Med. 2013;274(3):252-62.

35. Johnson AC, Stahl A, Zager RA. Triglyceride accumulation in injured renal tubular cells: alterations in both synthetic and catabolic pathways. Kidney Int. 2005;67(6):2196-209.

36. Vaziri ND. Causes of dysregulation of lipid metabolism in chronic renal failure. Semin Dial. 2009;22(6):644-51.

37. De Cosmo S, Menzaghi C, Prudente S, Trischitta V. Role of insulin resistance in kidney dysfunction: insights into the mechanism and epidemiological evidence. Nephrol Dial Transplant. 2013;28(1):29-36.

38. McLaughlin T, Abbasi F, Cheal K, Chu J, Lamendola C, Reaven G. Use of metabolic markers to identify overweight individuals who are insulin resistant. Ann Intern Med. 2003;139(10):802-9.

39. Jensen JS, Feldt-Rasmussen B, Strandgaard S, Schroll M, Borch-Johnsen K. Arterial hypertension, microalbuminuria, and risk of ischemic heart disease. Hypertension. 2000;35(4):898-903.
40. Bakker AJ. Detection of microalbuminuria. Receiver operating characteristic curve analysis favors albumin-to-creatinine ratio over albumin concentration. Diabetes Care. 1999;22(2):307-13.

41. Eknoyan $\mathrm{G}$, Hostetter $\mathrm{T}$, Bakris GL, Hebert L, Levey AS, Parving HH, et al. Proteinuria and other markers of chronic kidney disease: a position statement of the national kidney foundation (NKF) and the national institute of diabetes and digestive and kidney diseases (NIDDK). Am J Kidney Dis. 2003:42(4):617-22.

42. Salinero-Fort MA, San Andres-Rebollo FJ, de Burgos-Lunar C, GomezCampelo P, Chico-Moraleja RM, Lopez de Andres A, et al. Five-year incidence of chronic kidney disease (stage 3-5) and associated risk factors in a Spanish cohort: the MADIABETES Study. PLoS One. 2015;10(4), e0122030.

\section{Submit your next manuscript to BioMed Central and take full advantage of:}

- Convenient online submission

- Thorough peer review

- No space constraints or color figure charges

- Immediate publication on acceptance

- Inclusion in PubMed, CAS, Scopus and Google Scholar

- Research which is freely available for redistribution 